\title{
Methods to Test the "Dimming Effect" Produced by a Decrease in the Number of Photons Received from Receding Light Sources
}

\section{Zilberman ( $\square$ mzilberman137@gmail.com ) \\ Shiny World Corp.}

\section{Research Article}

Keywords: Dimming effect, Doppler effect, Standard Candles, photon, time dilation

Posted Date: December 31st, 2020

DOI: https://doi.org/10.21203/rs.3.rs-138372/v1

License: (c) (1) This work is licensed under a Creative Commons Attribution 4.0 International License.

Read Full License 


\title{
Methods to Test the "Dimming Effect" Produced by a Decrease in the Number of Photons Received from Receding Light Sources
}

\author{
M. Zilberman, M.Sc.* \\ Shiny World Corp., Canada
}

\begin{abstract}
The hypothetical "Dimming Effect" describes the change of the number of photons arriving from a moving light source per unit of time. In non-relativistic systems, the "Dimming effect" may occur due to the growing distance of light sources moving away from the receiver. This means that due to the growing distance, the photons continuously require more time to reach the receiver, which reduces the number of received photons per time unit compared to the number of emitted photons.
\end{abstract}

Understandably, the proposed "Dimming effect" must be tested (confirmed or rejected) through observations. a. This article provides the formula for the calculation of "Dimming effect" values using the redshift parameter Z widely used in astronomy.

b. The "Dimming effect" can possibly be detected utilizing the orbital movement of the Earth around the Sun. In accordance to the "Dimming effect", observers on Earth will view 1.0001 more photons per time unit emitted by stars located near the ecliptic plane in the direction of the Earth orbiting the Sun. And, in contrast, observers will view only 0.9999 photons per time unit emitted by stars located near the ecliptic plane in the direction opposite to the Earth orbiting the Sun. Calculating precise measurements of the same stars within a 6-month period can possibly detect this difference. These changes in brightness are not only for specific stars, as the change in brightness takes place for all stars near the ecliptic in the direction of the Earth's orbit around the Sun and in the opposite direction.

c. The "Dimming effect" can possibly be detected in a physics laboratory using a moving light source (or mirror) and photon counters located in the direction of travel and in the opposite direction.

d. In theory, Dilation of time can also be used for testing the existence of the "Dimming effect." However, in experiments on Earth this effect appears in only the $14^{\text {th }}$ digit after the decimal point and testing does not appear to be feasible.

e. Why is it important to test the "Dimming effect?" If confirmed, it would allow astronomers to adjust values of "Standard Candles" used in astronomy. Since "Standard Candles" are critical in various cosmological models, the "Dimming effect" can correct models and/or reveal and support new models. If it is proved that the "Dimming effect" does not exist, it will mean that the number of photons arriving per unit of time does not depend on the speed of the light source and observer, which is not so apparent.

Keywords: Dimming effect, Doppler effect, Standard Candles, photon, time dilation

The hypothetical "Dimming effect" disclosed in article [1] is similar to the Doppler effect, in which wavelength and frequency of photons hitting the light detector depends on the speed of the light source. The "Dimming effect" describes however, not the change of frequency of individual photons but a change in the number of photons arriving per time unit. In nonrelativistic systems, the "Dimming effect" occurs due to the fact that as light sources move away, the distance between the emitter and the receiver constantly increases, and the photons always take longer to reach the receiver. This reduces the number of photons received per time unit compared to the number of emitted photons.

The faster the light source moves away from us, the fewer photons hit the detector per unit of time. The model (emission of photons from an object moving away from the observer)

*email address: MZilberman137@gmail.com 
presented in [1] is similar to the models presented in [2] and [3]. However, an important difference is that research [1] focused on changes in the number of incoming photons per time unit, while research [2] and [3] focused on changes in the wavelength of incoming photons. Clearly, this hypothetical "Dimming Effect" must be tested (confirmed or rejected) through the course of observations.

\section{Simple real-life illustration}

Noted, we can observe a type of "Dimming effect" in real-life multiple times daily. If during hand-washing, we move our palms toward the faucet (in fig. 1 the hands are moving from position A up to position B), the water fills our palms faster (while we are moving them up). If we are bringing our palms down from the faucet (in fig.2, the hands are moving from position A down to position B) the water fills our palms slower (while we are moving them down). As a result, the amount of water accumulated by distancing our palms on fig. $2 \mathrm{~B}$ is always less than amount of water accumulated in palms approaching to faucet on the fig. $1 \mathrm{~B}$.

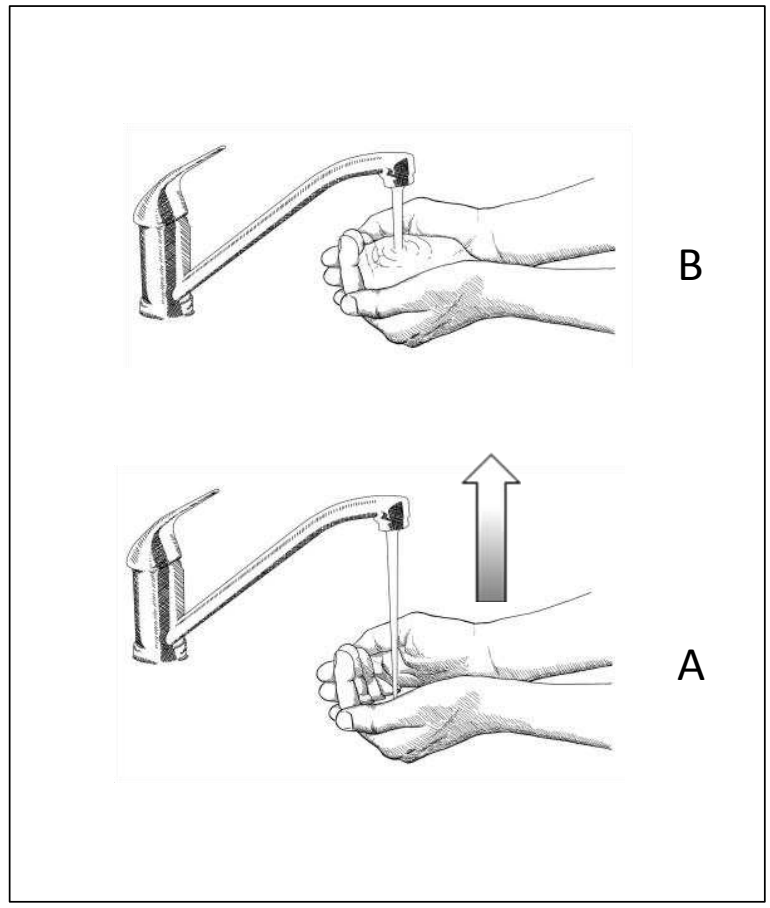

Fig.1. Folded palms are moving toward faucet.

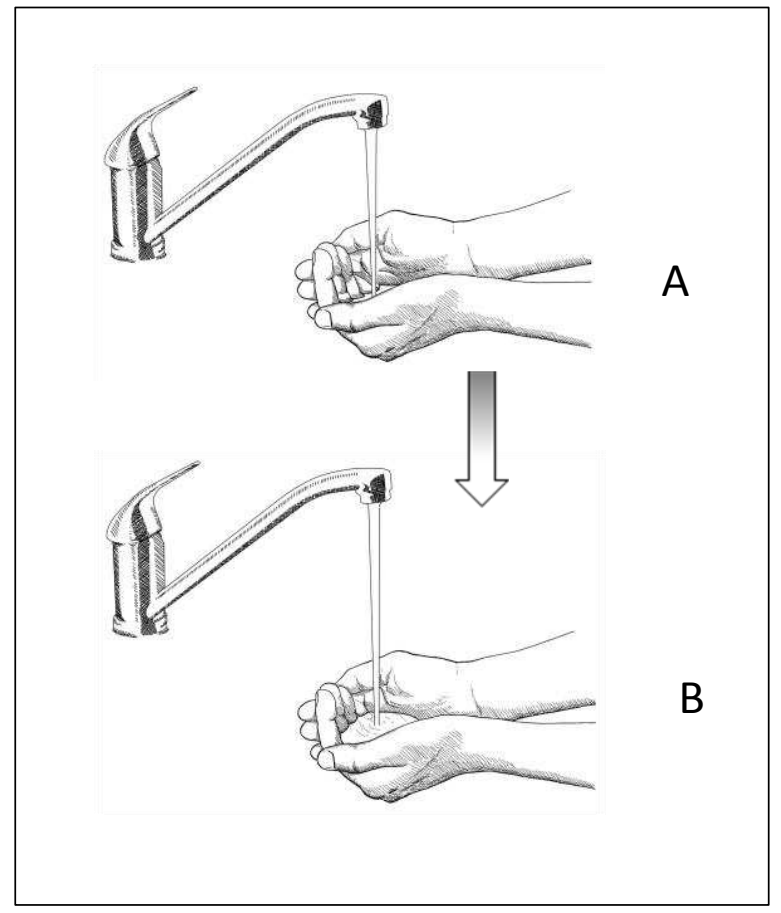

Fig.2. Folded palms are distancing from faucet.

If in the above model we replace the stream of water in figs. 1 and 2 with a beam of photons and replace our hands with a photon detector (e.g. a telescope) we can expect a decrease of detected photons (per time unit) arriving from distancing object and increase the number of detected photons (per time unit) arriving from approaching objects.

Of course, this is an oversimplified model. Photons are not only quanta of light but also waves. Therefore, it is not necessary that the simplified model presented above correctly describes the counting of photons emitted by distancing objects. There is good chance that a number of photons arriving from moving sources (per time unit) is independent from the relative speed of 
the emitter and receiver of photons. However, since there is no clear answer to this question and since the hypothetical "Dimming effect" can significantly influence the calculation of the "Standard Candles" in astronomy, it makes sense to test the existence of the "Dimming effect" in a physics laboratory or by using astronomical observations.

\section{The Dimming effect and $Z$ parameter}

Formula (1) for the calculation of the "Dimming Effect" was obtained in article [1] as

$$
N / N_{0}=\frac{\sqrt{1-\frac{V^{2}}{c^{2}}}}{1+\frac{V}{c}}
$$

Here $N_{0}$ is the number of emitted photons, $N$ is the number of observed photons, $V$ is the speed of the light source and $c$ is the speed of light.

First, let us rewrite equation (1) using the widely accepted parameter Z, which describes redshift. It is defined as

$$
Z=\frac{\lambda-\lambda_{0}}{\lambda_{0}}
$$

where $\lambda_{0}$ is the wavelength of emitted photons and $\lambda$ is the wavelength of received photons. Using the equation $\lambda^{*} \nu=c$ (where $v$ is the frequency of light and $c$ is the speed of light in a vacuum) and the Doppler effect formula (3) from the book [4]

$$
v=v_{0} \cdot \frac{\sqrt{1-\frac{V^{2}}{c^{2}}}}{1-\frac{V}{c} \cdot \cos \theta}
$$

(where $v_{0}$ is the radiation frequency at the source of light, $\nu$ is the observed radiation frequency, $V$ is the speed of the source relative to the observer and $\theta$ is the angle between the direction to the source and the velocity vector in the receiver's frame of reference) for $\theta=\pi$ we obtain formulas (4) and (5)

$$
v=v_{0} \cdot \frac{\sqrt{1-\frac{V^{2}}{c^{2}}}}{1+\frac{V}{c}} \quad \text { (4) } \quad \lambda=\lambda_{0} \cdot \frac{1+\frac{V}{c}}{\sqrt{1-\frac{V^{2}}{c^{2}}}}
$$

From formulas (2) and (5) we likewise infer

$$
Z+1=\frac{\lambda}{\lambda_{0}}=\frac{1+\frac{V}{C}}{\sqrt{1-\frac{V^{2}}{c^{2}}}}
$$


Now in formula (1) we can replace part

$$
\begin{gathered}
\frac{\sqrt{1-\frac{V^{2}}{c^{2}}}}{1+\frac{V}{C}} \text { with } \frac{1}{Z+1} \text { and rewrite the Dimming effect formula using } Z \text { as } \\
N / N_{0}=\frac{1}{Z+1}
\end{gathered}
$$

Two comments to formula (6)

a. Using formula (6) we must remember the condition $\theta=\pi$ (the light source is moving away from us), which we used above. For distant galaxies, this is almost always the case due to the expansion of the Universe.

b. Formula (6) was derived from formula (1), which was calculated within the framework of Special Relativity. General Relativity calculates the $Z$ parameter using the curvature of spacetime. Cosmology uses the $Z$ parameter in models with cosmological expansion. In these cases, the relationship between $N / N_{0}$ and $Z$ can be obtained in further complex calculations, which are beyond the scope of this article.

Experiments to test the existence of the Dimming effect

1. Astronomy

As it was mentioned in [1] in accordance to formula (1) the "Dimming effect" is negligible for light sources with low speed. Per example taken from [1], if 2 observers are located at diametrically opposite points of the earth's equator and attempt to measure the brightness of a distant object of light, located in the equatorial plane, the difference in brightness will only be

$$
460(\mathrm{~m} / \mathrm{s}) * 2 / 300,000,000(\mathrm{~m} / \mathrm{s})=3.06667 \mathrm{E}-06,
$$

where $460 \mathrm{~m} / \mathrm{s}$ is the Earth's rotating speed at the equator.

However, the "Dimming effect" can possibly be detected using the orbit of the Earth around the Sun (Fig. 3).

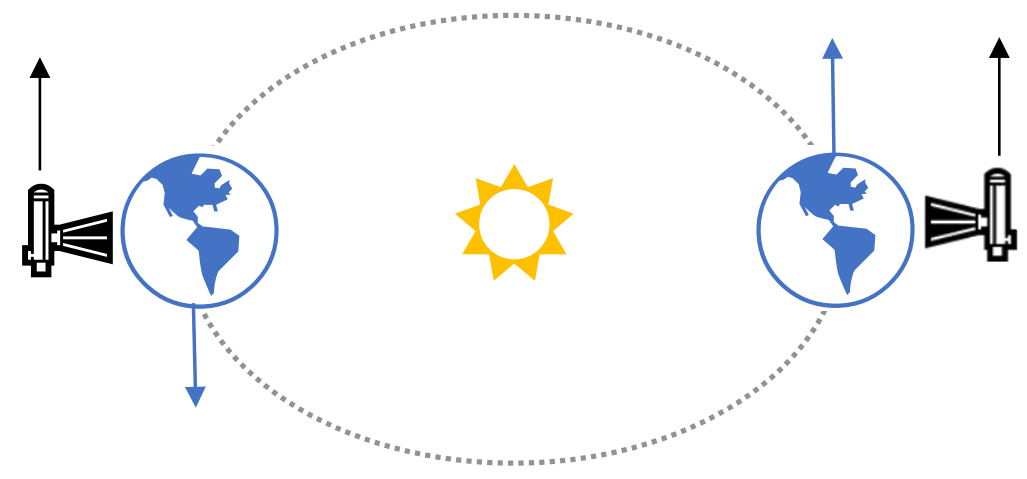

Fig.3 Model of "Dimming effect" test using the Earth's orbit around the Sun. 
Since the speed of the Earth's orbit around the Sun is approximately $30 \mathrm{~km} / \mathrm{s}$, telescopes aimed in the direction of the Earth's orbit will receive slightly more photons per second, while telescopes aimed in the direction opposite to the Earth's orbit will receive slightly less photons per second. According to formula (1), this ratio is approximately

$$
\frac{N}{N_{0}}=\frac{\sqrt{1-\frac{30^{2}}{300000^{2}}}}{1-\frac{30}{300000}}=1.00010
$$

for stars located near the ecliptic plane in the direction of the Earth's orbit around the Sun and is about

$$
\frac{N}{N_{0}}=\frac{\sqrt{1-\frac{30^{2}}{300000^{2}}}}{1+\frac{30}{300000}}=0.99990
$$

for the stars located near the ecliptic plane in the direction opposite to Earth's orbit around the Sun.

It is not known if current telescopes can detect such a small change in brightness. Fortunately, however, these changes in brightness are not only for specific stars. All stars near the ecliptic plane in the direction of the Earth's orbit around the Sun will appear 1.0001 brighter while in the opposite direction - will appear 0.9999 dimmer. Perhaps this will assist to detect the effect.

Reference stars for differential brightness measurement should be located at an angle of $90^{\circ}$ to the ecliptic plane. For these stars, the nonrelativistic "Dimming effect" is zero, and the speed of $30 \mathrm{~km} / \mathrm{s}$ is too low to observe the transverse "Dimming effect" (similar to the transverse Doppler effect).

\section{Physics}

The simplest method to test the "Dimming effect" is likely using a light source that travels to and from a photon counter. In fig. 4 light source (1) moves between photon counters 2 and 3. As the light source approaches photon counters $3 \mathrm{a}$ and $2 \mathrm{~b}$ and moves away from counters $2 \mathrm{a}$ and $3 \mathrm{~b}$, the "Dimming effect" predicts that photon counters $3 \mathrm{a}$ and $2 \mathrm{~b}$ will count more photons than counters $2 \mathrm{a}$ and $3 \mathrm{~b}$ per second.

After a certain amount of time the detectors may accumulate enough photons to detect a difference or to confirm that there is no difference in the brightness of approaching and receding light sources. These light sources can possibly be simple mirrors that reflect the light of the static light sources.

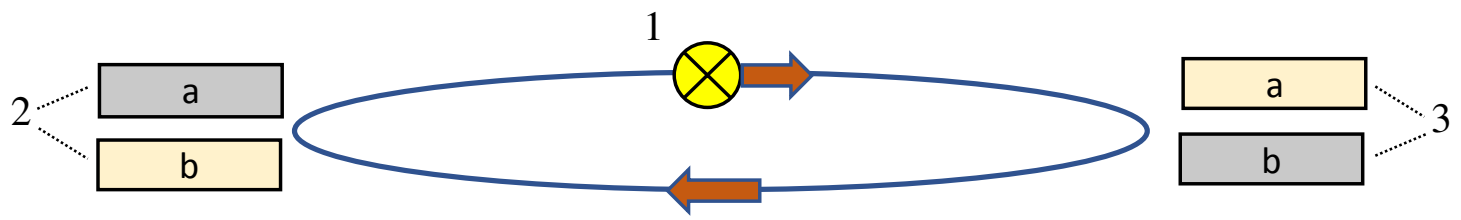

Fig.4. Testing of the Dimming effect in a laboratory. Light source 1 is moving between light detectors 2 and 3 . 
The exact shift in the number of detected photons is dependent on the speed of light source 1 and is determined by formula (1).

Two items to consider

a. The distance between the light source and the photon counters should be optimally large enough to minimize the effect of the inverse square law on brightness changes.

b. If possible, it would make sense to elongate the trajectory of the light source and make it look as a stretched ellipse rather than a circle. In another method of the experiment, the light source (or mirror) moves left and right along a straight line and the photon counters separately count the number of photons arriving from an approaching and receding source. Per [1] in non-relativistic systems the "Dimming effect" occurs due to moving away light sources the distance between the emitter and receiver constantly increases and photons require additional time to reach the receiver. This reduces the number of receiving photons per time unit compared to the number of emitted photons.

3. Time dilation and the "Dimming effect"

In the section above, "The Dimming effect and $Z$ parameter" we obtained the relationship between the dimming of objects and the redshift parameter $Z$ as

$$
N / N_{0}=\frac{1}{Z+1}
$$

This formula was obtained within the framework of Special Relativity. However, there are at least two other mechanisms that also produce redshift. These mechanisms are gravity and cosmological expansion. The gravitational shift of radiated frequencies was confirmed through experiment in 1959 by R.V. Pound and G.A. Rebka [5]. In theory, we could utilize the identical experimental setup to test whether the number of received photons per time unit depends on the gravitational potential of the location of the photon emitter.

Illustration fig. 5 below shows the portrayal of such an experiment. The photon counter (3) is located on the Earth's surface (1), and the light source (2) is located above the Earth's surface. In this configuration, light undergoes a blue shift as (as proven in [5]) on the Earth's surface (1) time is slightly dilated compared to the location of the source (2). In this case $\mathrm{Z}$ is negative and we can expect $N / N_{0}>1$. (Indeed, the number of photons received per time unit in the receiver's reference frame will be greater than the number of photons emitted per time unit in the emitter's reference frame.)

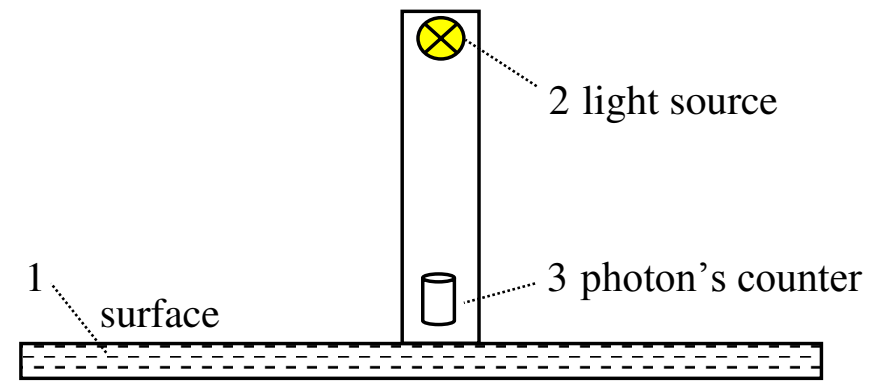

Fig.5. Testing of the "Dimming effect" in a laboratory. Light source 2 is above the light detector 3. 
Unfortunately, the "Dimming effect" in this experimental setup is so weak so that it is likely impossible to detect.

Per calculations provided in [5],

$$
v_{\mathrm{h}}-v_{0}=v_{0} \cdot g \cdot h \cdot / \mathrm{c}^{2} \cdot(1+\mathrm{h} / \mathrm{R}) \approx v_{0} \cdot \mathrm{h} \cdot\left(1.09 \cdot 10^{-18}\right)
$$

where $v_{h}$ is the frequency of the emitter at height $h, v_{0}$ is the frequency at the surface, $g$ is Newton's Gravitational constant, $\mathrm{c}$ is the speed of light, $\mathrm{R}$ is the radius of the Earth and $\mathrm{h}$ is the altitude measured in $\mathrm{cm}$.

Thus, even with an altitude of $100 \mathrm{~m}$ (i.e. $10,000 \mathrm{~cm}$ ), modern equipment likely will not be able to detect the shift in brightness located in the $14^{\text {th }}$ digit after the decimal point.

\section{Conclusion}

The "Dimming effect" is very weak for speeds incomparable to the speed of light $c$. As such it does not attract scientific attention as some inexplainable physics phenomenon. However, theoretical analysis of the number of photons arriving per time unit of a moving light source indicates to the existence of this effect and allows us to calculate its strength. Depending on the precision of equipment the "Dimming effect" may be detected in astronomical observations and in a physics laboratory.

Why is testing of the "Dimming effect" important? If its existence is confirmed, this will allow us to adjust the values of "Standard Candles" used in astronomy for sources at a speed comparable to the speed of light. Since values of "Standard Candles" are critical in various cosmological models, the "Dimming effect" may correct certain models and/or reveal and support new models.

On the other hand, if it is experimentally proven that the "Dimming effect" does not exist, this will also provide important information. It will mean that the number of photons arriving per time unit does not depend on the source or detector. i.e. it does not matter whether the light source is approaching or moving away - the number of arriving photons per time unit remains unchanged. Generally speaking, this is not so apparent.

The author is grateful to V. Yershov for valuable comments and discussions.

\section{References}

1. Zilberman M. The "Dimming Effect" Produced by the Application of Doppler Effect on the Quantity of Photons Arriving to a Receiver and its Implication to Astronomy. Intellectual Archive, v.9, \#3, 1-5 (2020). https://DOI.org/10.32370/IA_2020_09_1.

2. Klacka, J. \& Saniga, M. Doppler effect and nature of light. Earth, Moon, and Planets 59, 219-227 (1992). https://doi.org/10.1007/BF00054054

3. Tiwari S.C. Doppler effect and frequency-shift in optics. https://arxiv.org/abs/quant$\mathrm{ph} / 0410084$ (2006) 
4. Landau L. D., Lifshits E. M. Teoriia polia. - Izdanie 7-e, ispravlennoe. [Theory of the field. - 7th edition, revised] (Nauka, Moscow, 1988). - Pp. 158-159. - (Teoreticheskaia fizika, tom II ["Theoretical Physics", volume II]). — ISBN 5-02-014420-7.

5. Pound R. V., Rebka, Jr, G. A. Gravitational Red-Shift in Nuclear Resonance. Physical Review Letters 3, 439-441. https://DOI.org/10.1103/PhysRevLett.3.439 (1959)

\section{Competing interests}

The author declares no competing interests 
Figures

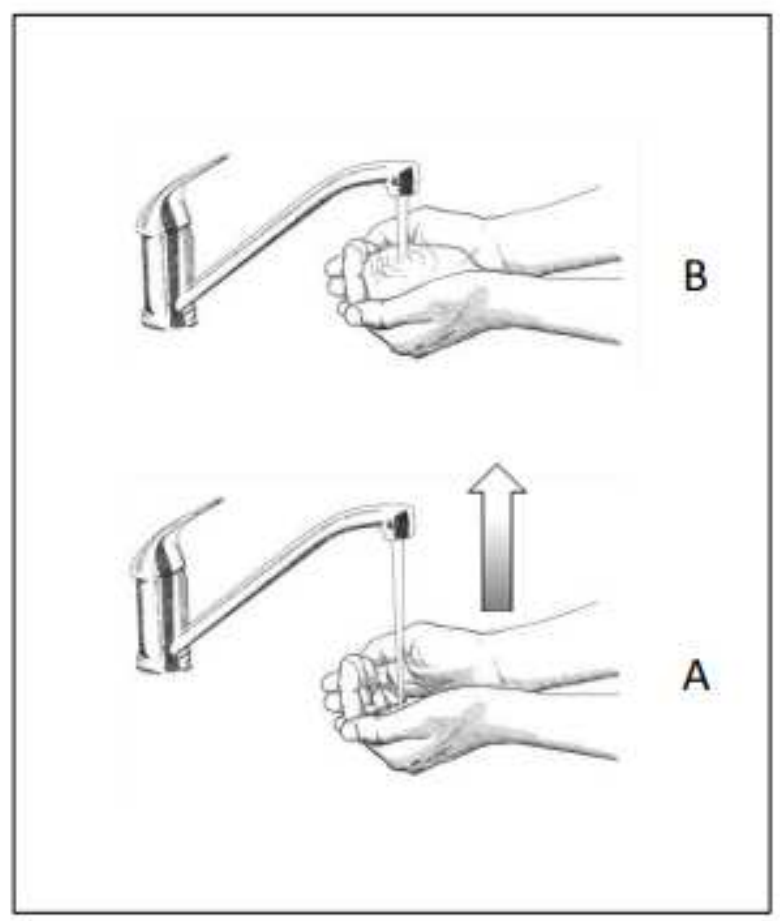

Figure 1

Folded palms are moving toward faucet.

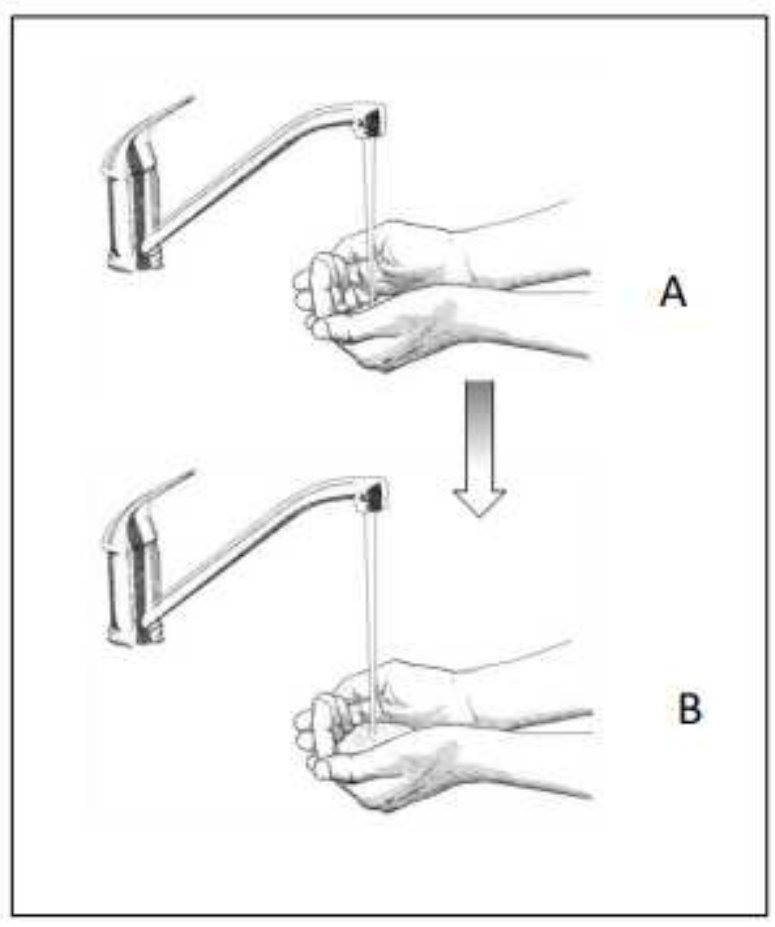

Figure 2

Folded palms are distancing from faucet. 


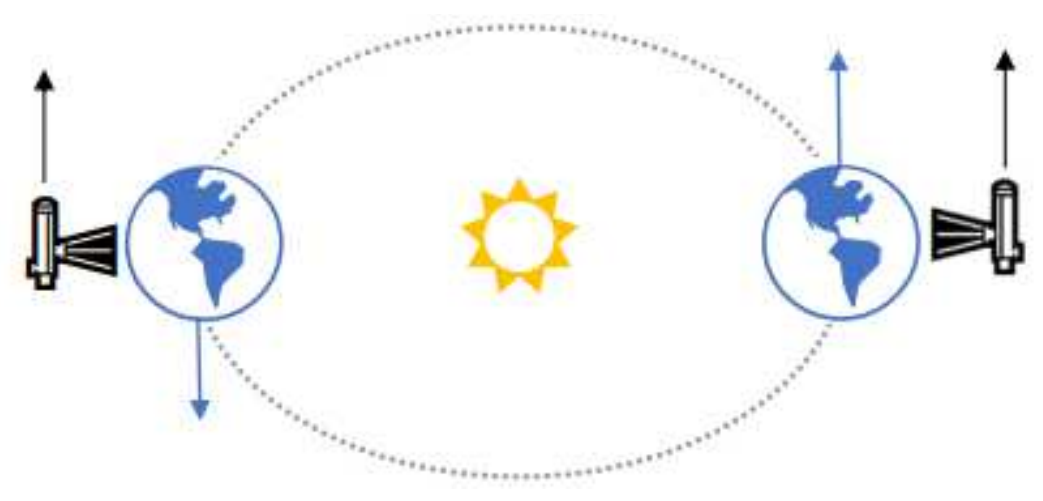

Figure 3

Model of "Dimming effect" test using the Earth's orbit around the Sun

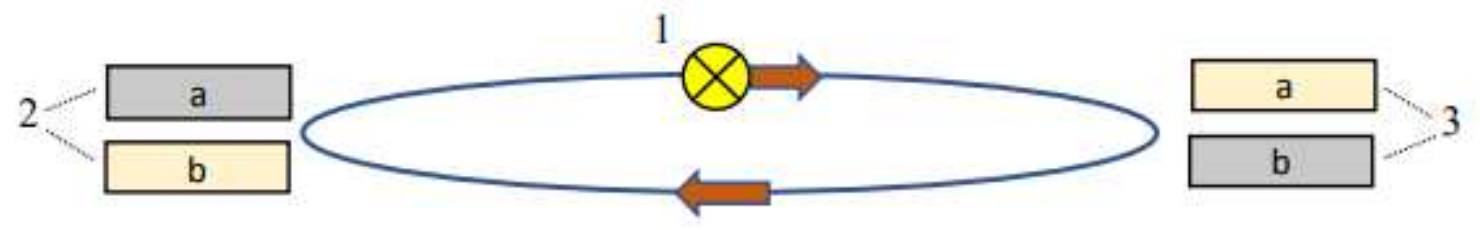

\section{Figure 4}

Testing of the Dimming effect in a laboratory. Light source 1 is moving between light detectors 2 and 3 .

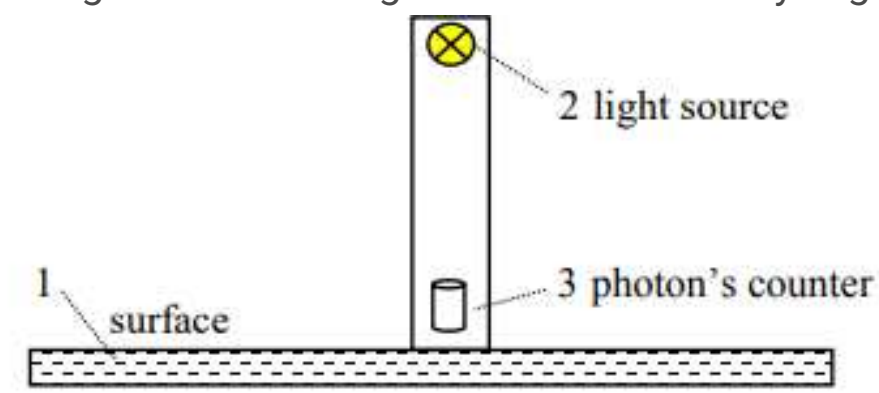

\section{Figure 5}

Testing of the "Dimming effect" in a laboratory. Light source 2 is above the light detector 3 